\title{
Los niveles de la investigación socioeducativa: lógica y relacionalidad investigativa
}

\author{
The levels of socio-educational research: \\ logic and relationality research
}

Luis Carlos Morales Zúñiga*

\section{RESUMEN}

Este escrito constituye un estudio sobre la lógica de la investigación socioeducativa, tomando en cuenta los elementos esenciales de la investigación, y las relaciones entre esos elementos, las cuales delinean un diseño investigativo para la indagación de los fenómenos educativos. En una primera parte se analizan los niveles formales de la investigación socioeducativa, con especial énfasis en los planos filosófico, científico y técnico de la lógica investigativa. Posteriormente se propone el concepto de relacionalidad como un enfoque articulador para abordar la lógica de investigación, y establecer una concepción integradora de los distintos niveles que componen el proceso investigativo, así como una postura investigativa basada en la concepción relacional de la educación.

Palabras clave: Investigación educativa, filosofía, ciencia, técnicas de investigación, relacionalidad, educación

\begin{abstract}
This paper is a study on socio-educational research logic, focusing on the essential elements of research designs, but especially on the relations between those elements, which denote the most important requirements for the research design in the inquiry about educational phenomena. In the first part we discuss the formal levels of socio-educational research, with special emphasis on the philosophical, scientific and technical elements of research logic. Subsequently, we propose the concept of relationality as a coordinating approach to address the logic of research, and establish an integrated approach of the different levels that make up the research process and also a research framework based on the relational conception of educational phenomenon.
\end{abstract}

Key words: Educational research, philosophy, science, research techniques, relationality, education

\section{INTRODUCCIÓN}

El proceso de investigación en las ciencias sociales en general, y en las ciencias de la educación en particular, posee características propias y, sobre todo, una lógica que da sentido al trabajo de indagación sobre la realidad social y socioeducativa. No se trata, como podría pensarse, de la simple aplicación del método científico, para obtener como resultado una investigación científica,

* Departamento de Educación Secundaria, Facultad de Educación, Universidad de Costa Rica; luis23m@gmail.com. 
pues al limitarnos al plano técnico que la palabra aplicación connota, es posible suponer que se trata de la sistematización de una serie de pasos y su consecuente puesta en práctica, lo cual sería demasiado simple.

Sin embargo, como lo había planteado Pierre Bourdieu (1994), "las etapas de la investigación no son cronológicas, sino que son lógicas", lo que precisamente confirma el hecho de que en un proceso investigativo, lo importante no es seguir una especie de recetario teórico-metodológico (tal como lo muestran o como sugieren los tratados de investigación al señalar una serie de pasos que van desde el planteamiento del tema hasta la presentación del informe, pasos tan importantes como obvios, al estilo Sampieri (1998), sino que se trata de establecer relaciones lógicas entre las partes y las etapas que integran la investigación.

Esta idea general es la motivación particular de este trabajo, el estudio de las partes que componen una investigación socioeducativa y, sobre todo, el estudio de las relaciones entre esas partes, que facultan al diseño de investigación para funcionar lógica y coherentemente como un sistema dotado de capacidad explicativa, interpretativa y de aplicación.

La investigación socioeducativa abarca, desde nuestro punto de vista, varios elementos fundamentales que es necesario aclarar. Partimos de un argumento base, la educación es, necesariamente, un fenómeno social; este argumento no se trata de un juicio de sentido común, sino de un intento de clarificación. Al decir que la educación es un fenómeno social, se quiere poner en claro el ámbito epistemológico del fenómeno educativo, esto es, un esfuerzo por definir los límites de la explicación y el conocimiento, y la validez del conocimiento que se construye sobre la educación. Durkheim (1997) ha declarado que "lo social se explica por lo social", es decir, que solo en sociedad y mediante los recursos del conocimiento social y científico social, podemos dar cuenta de los fenómenos sociales, entre ellos la educación.

Por otro lado, asumir que la educación es un fenómeno social, quiere decir que todos los fenómenos que a veces se hacen pasar por cuestiones meramente técnicas, tales como las estrategias didácticas, los materiales didácticos, el currículum y los diseños curriculares, las metodologías de enseñanza, así como 
todos los fenómenos, las prácticas y los valores implicados en la educación, son inminentemente sociales, y deben ser conocidos científico-socialmente.

Otra inferencia importante que podemos obtener del hecho de considerar la educación como un fenómeno social, es que la investigación educativa es, entonces, investigación social, pero sobre todo investigación científico social. Como una de las formas de la investigación social, cuenta con los mismos retos, los mismos problemas, así como las mismas posibilidades teóricas y metodológicas que comparten todas las ciencias sociales. En este ensayo se busca dar cuenta de los niveles filosófico, científico, técnico, así como del enfoque de relacionalidad, el cual se propone como una forma de abordar la investigación educativa, que permite producir y reproducir relaciones analíticas, hermenéuticas y reflexivas sobre los fenómenos educativos estudiados con perspectiva científica.

\section{LOS NIVELES FORMALES DE LA INVESTIGACIÓN (SOCIOEDUCATIVA): FILOSOFÍA, CIENCIA Y TÉCNICA}

La historia del pensamiento occidental, según ha sido observado por diversos autores entre los cuales Constantino Láscaris (1976), Max Weber (1979) o Werner Jaeger (1962) son solo algunos ilustres ejemplos, deviene de la filosofía a la ciencia, y de la ciencia a la técnica. Va desde el conocimiento filosófico hacia el conocimiento técnico, pasando por el conocimiento científico. Esto es lo mismo que decir: el conocimiento va de la libre especulación, a la verificación empírica (el conocimiento de algunas certidumbres o al menos de algunas constantes en el desarrollo de los fenómenos de la realidad), y luego hacia el aprovechamiento de ese conocimiento traducido en herramientas, o técnicas, que permiten dar cuenta, captar o incidir sobre la realidad. Aunque estas tres palabras centrales representan el devenir ya conocido del desarrollo del pensamiento, no es posible eludirlas como punto de partida esencial.

Cada uno de estos tres niveles está implicado en el proceso de investigación, pues la investigación es el trabajo de ir en busca del conocimiento razonable, verificable, y con implicaciones prácti- 
cas. En esa búsqueda es necesario pensar, conocer, aplicar, y llegar a resultados. Constantino Láscaris (1976) ha dicho que investigar es cazar y desarrolla una analogía entre el cazador y el investigador. Tanto la palabra vestigio como la de investigar provienen de la voz latina invadere, que significa según Corominas "penetrar violentamente en alguna parte” (1983: 339). El cazador y el investigador van en busca de vestigios, y penetran en el campo, en la realidad por causa de su búsqueda. Ambos deben pensar, reflexionar, concebir una teoría sobre lo que buscan, luego conocer o verificar empíricamente las reflexiones hechas en cuanto al objeto de su búsqueda (el animal o la respuesta a un problema), desarrollar y utilizar en la práctica una serie de técnicas (de caza o de investigación) y, finalmente, dice Láscaris, inmolar al animal, alcanzar una respuesta al problema, y por ende, una satisfacción pragmática, aunque temporal.

Así como en la caza, en la investigación los problemas son momentáneos. Un cazador logra, mediante los resultados de su trabajo, una respuesta a una necesidad momentánea, circunstancial -podría haber cazado otro tipo de animal, con otro tipo de técnicas, para otros fines, si estuviera en otro tiempo y en otras tierras- y, además, con la inmolación del animal, el ciclo inicia nuevamente. También el investigador, al terminar su trabajo, resuelve o da respuesta a un problema de manera momentánea y circunstancial, sus respuestas son apenas pasajeras, efímeras, y eventualmente inútiles en el futuro, pues serán superadas. Su trabajo ha servido no para dar respuestas definitivas, y al igual que el cazador, debe comenzar de nuevo. Esto había sido observado ya por Nietzsche, cuando dijo:

...las verdades son ilusiones de las que se ha olvidado que lo son, metáforas que se han vuelto gastadas y sin fuerza sensible, monedas que han perdido su troquelado y no son ahora consideradas como monedas, sino como metal (1980: 6).

Si bien las respuestas a las que se llega en un proceso de investigación tienden a ser momentáneas, el proceso para alcanzar esas respuestas se puede mejorar para producir cada vez mejores resultados. Así como el cazador, conforme adquiere más expe- 
riencia en su oficio, afina sus técnicas, sus astucias y sus armas, el investigador se hace en la investigación y mejora en cuanto más practique su oficio. En ambos casos, la experiencia es un aspecto fundamental, así como el aprendizaje de lo que se ha hecho antes, de los métodos y el conocimiento acumulado hasta el momento. Esta analogía entre la caza y la investigación sugerida por Constantino Láscaris es tan consistente como precisa, tanto que podríamos seguir enumerando una larga lista de rasgos análogos entre ambos oficios, además de los que ya ha mencionado Láscaris en su texto (1976), y lo que hemos agregado sobre lo efímero de ambas actividades.

Los niveles formales de la investigación educativa están constituidos entonces por lo filosófico, lo científico, y lo técnico, lo que nos interesa es especificar ahora, en qué sentido se aprecian o se manifiestan estos tres niveles en los procesos de investigación científica y educativa, los cuales son observables, desde nuestra perspectiva, de la siguiente manera:

\section{Nivel filosófico}

En cuanto a la filosofía, sabemos - da la impresión que desde siempre- que se trata de la unión de dos palabras griegas ( $\varphi \imath \lambda i \alpha$ ) philia/amor y $\Sigma o \varphi i \alpha / s o f i ́ a / s a b i d u r i ́ a)$, de manera que el filósofo, o la persona que hace filosofía, siente amor por el saber, y esto le lleva entonces a considerar todas las perspectivas y posibilidades, a cuestionar la realidad, y el conocimiento que se obtiene sobre ella, así como a cuestionar también el medio por el cual se obtiene el conocimiento, esto es, evaluar críticamente el aparato cognoscente.

Hacer filosofía es pensar con rigor, con método pero, sobre todo, pensar libremente. Parece contradictorio decir que se debe pensar con rigor y con libertad al mismo tiempo. Esto es posible en el tanto el acto del pensamiento se ejecuta siguiendo el rigor (no la rigidez) lógico y, al mismo tiempo, con la capacidad crítica de evaluar los resultados del acto de pensar, el método de ese acto, y otras formas de concebir la realidad, con énfasis en la no aceptación de ningún tipo de dogmatismo, político, religioso, ideológi- 
co ni científico. En resumen, pensar de manera amplia, rigurosa y no dogmática, es una de las formas más fecundas del filosofar.

Este nivel se ocupa, entonces, de pensar con rigor, pero con flexibilidad al mismo tiempo. La libertad de pensamiento frente al dogmatismo es una de las características fundamentales del pensamiento filosófico. En cuanto a la investigación, su aporte se manifiesta en tres subniveles.

\section{Subnivel ontológico}

La ontología se define, habitualmente, como el estudio del ser en cuanto es. Lo que es, al ser algo, es por lo tanto real, de manera que esta rama del conocimiento filosófico se encarga del estudio y definición de la realidad en cuanto es. En su obra Metafísica, Aristóteles habla de una ciencia que se encarga del estudio de lo "de lo que es en cuanto es", una ciencia universal, que se encarga del estudio de los principios fundamentales, según dice Aristóteles:

Hay una ciencia que estudia lo que es, en tanto que algo es, y los atributos que, por sí mismo, le pertenecen. Esta ciencia, por lo demás, no se identifica con ninguna de las denominadas particulares. Ninguna de las otras (ciencias), en efecto, se ocupa universalmente de lo que es, en tanto que algo es, sino que tras seccionar de ello una parte, estudia los accidentes de esta: así, por ejemplo, las ciencias matemáticas (1998: 161).

Siguiendo, entonces, a Aristóteles, los dominios de las ciencias son los accidentes de la realidad, sectores de lo real, pero no abarcan la realidad como fenómeno total. No obstante, en cada ciencia $y$, sobre todo, en cada forma de hacer ciencia, hay un principio de orden ontológico, una concepción o idea de qué y cómo es la realidad. La ciencia positiva por ejemplo, considera que la realidad es algo objetivo, externo al sujeto, cognoscible mediante la experimentación y, sobre todo, representable tal cual es. El naturalismo científico, en cambio, no postula la objetividad como un principio constitutivo de la realidad, sino como una ficción del sujeto. Así, el naturalismo no buscará la separación del sujeto respecto del objeto; por el contrario, se interesará en las particularidades que el punto de vista del sujeto ańade a la realidad en estudio. 
La importancia que tiene la ontología para la investigación consiste en el hecho de que cualquier proceso investigativo busca dar cuenta de una parte de la realidad y esto implica, necesariamente, una concepción sobre esa realidad. Si pensamos que la realidad es objetiva o, por el contrario, que no lo es, si pensamos que la realidad es dinámica y dialéctica, o si creemos que la realidad es una ilusión, esto tendrá consecuencias directas en nuestros paradigmas de investigación, en los métodos investigativos, en el enfoque y hasta en los instrumentos mediante los cuales se captará tal o cual parte de lo real. Además, una posición sobre la realidad definirá también qué se puede estudiar y qué no se puede estudiar desde el punto de vista científico. Por ejemplo, en Las Reglas del método sociológico, Durkheim (1997) ha señalado que para contar con la existencia de los hechos sociales, es decir los datos del científico social, no solo es necesario que estos hechos sean externos al sujeto y coercitivos sobre él; se requiere, además, que sean generales al colectivo que estemos estudiando: lo que no es general no es un hecho social, y al no serlo, no es susceptible de ser estudiado científicamente.

Durkheim tiene, claramente, una posición ontológica, considera que la realidad es objetiva, y la representación que hacemos de esa realidad debe ser también objetiva, por ello ha recomendado tratar los hechos sociales como cosas, y alejarse sistemáticamente de las prenociones, lo que significa tomar distancia respecto de la propia subjetividad (Durkheim, 1997). Para la investigación socioeducativa, igualmente, la ontología aporta una concepción sobre la realidad de la cual forman parte los hechos educativos, y de acuerdo con nuestra concepción sobre la realidad, tendremos también implicaciones en nuestra forma de percepción de la realidad educativa.

\section{Subnivel epistemológico}

Es preciso señalar que el estudio sobre nuestra forma de conocer, sobre los mecanismos y métodos que utilizamos en el proceso de construcción de conocimiento, así como el estudio de la validez de esos procedimientos, es el dominio de la rama filosófica conocida como epistemología. En el campo investigativo, la epis- 
temología ocupa un lugar privilegiado, debido a que el proceso de investigación científica es uno de los procedimientos que más aceptación tiene como forma de construcción de conocimientos válidos, aunque ciertamente momentáneos. Claro es que hay otros mecanismos de producción de conocimiento, el arte, la literatura, el periodismo, incluso la inspiración, las revelaciones, la astrología o las visiones, son mecanismos considerados válidos e incluso normales por distintas sociedades en diferentes épocas. Aunque en nuestra sociedad, y en nuestra época, la investigación científica coexiste con todos los otros mecanismos de producción de conocimiento citados, y probablemente con muchos otros, la ciencia tiene un lugar central en la construcción de conocimiento válido. Tan importante es el conocimiento científico que Ernesto Sábato considera la ciencia como uno de los peores dogmas de nuestro tiempo:

Y así nos encontramos con un hecho curioso: los siglos XVIII y XIX desencadenaron una especie particularmente peligrosa de dogmatismo: el científico. Es cierto que en nuestro siglo, algunos de los más grandes epistemólogos han recomendado la cautela y la modestia; pero el hombre de la calle, impresionado por el desarrollo de la técnica, no ve esos titubeos teóricos y ha adquirido la más singular de las supersticiones: la de la ciencia; que es como decir que ha adquirido la superstición de que no debe ser supersticioso (1945: 20).

Este hecho particular, observado por Sábato, debe su existencia a la capacidad que ha tenido el conocimiento científico en producir, mediante la investigación, conocimientos que tienen fundamentación teórica y empírica; es decir, que son verificables mediante métodos diversos. Pero podemos ir aún más lejos. La investigación científica tiene también la capacidad de auto-cuestionamiento y auto-evaluación de sus propios procesos y lógicas internas, lo cual le ha permitido avanzar, superarse a sí misma, y sobre todo desechar conocimientos, con el convencimiento de que no hay necesariamente un conocimiento absoluto. Claro que hay ramas de la ciencia, o con mayor precisión, científicos, que no piensan así, que son dogmáticos, sin embargo, parafraseando a Sábato (1945), el pensamiento científico, el genuino, es una escuela de modestia, no 
hay quien no haya errado, y no hay dogma que no haya caído ante nuevos hechos y nuevas formas de hacer ciencia.

En este punto la epistemología se vuelve una rama fundamental, porque permite realizar dos procesos verdaderamente significativos para el desarrollo de la investigación científica. El primero, evaluar la validez de nuestros conocimientos, del conocimiento existente desarrollado por otros, y la validez de nuestros métodos, instrumentos, así como de nuestra forma de definir, captar y analizar los datos de la realidad. Por otra parte, la epistemología permite construir objetos de estudio, entendidos como sistemas de relaciones entre fenómenos, conceptos, variables y hechos. El punto de vista epistemológico es la partida, pues sobre todo en ciencias sociales, y claramente en la investigación socioeducativa, los objetos de estudio no están dados, no son cosas como quiere Durkheim (1997), sino que debemos tratarlos como cosas y construirlos.

Las cosas físicas están dadas, son un datum, ${ }^{I}$ y en el caso de la investigación científica, se trabaja necesariamente con datos, con cosas que le han sido dadas a nuestros sentidos, y a nuestros aparatos cognoscentes, y cuando estamos en el campo de la investigación científico social, las cosas no están dadas, no son objetos, sustancias, o tejidos, sino que se trata de dinámicas, relaciones, sentidos, ideas, formas de hacer, pensar y de actuar con respecto a diversos fenómenos, esto tiene la implicación de que los objetos de estudio deben ser construidos por el sujeto que investiga. Lo anterior no quiere decir que las ciencias naturales no tengan un nivel epistemológico en su investigación, el investigador del mundo natural también debe construir relaciones entre fenómenos, la diferencia está en que esos fenómenos existen en forma concreta, pero otros, como el Estado, el aprendizaje, el conocimiento, los valores, son abstracciones que, en el mejor de los casos, se vuelven observables mediante las prácticas de los individuos, sus conductas y sus representaciones.

La epistemología sirve, entonces, como forma de evaluación del conocimiento, de su validez, y de los medios que utilizamos

\footnotetext{
${ }^{1}$ La voz latina datum, de donde procede el vocablo castellano dato, es el pasado del verbo dare, y entonces significa algo que nos ha sido dado, una cosa dada.
} 
para construirlo, pero sobre todo sirve para construir objetos de estudio. Gastón Bachelard ha dicho sobre este problema:

Ante todo, es necesario saber plantear los problemas. Y dígase lo que se quiera, en la vida científica los problemas no se plantean por sí mismos. Es precisamente este sentido del problema el que indica el verdadero espíritu científico. Para un espíritu científico todo conocimiento es una respuesta a una pregunta. Si no hubo pregunta, no puede haber conocimiento científico. Nada es espontáneo. Nada está dado. Todo se construye (2000: 16).

Los procedimientos específicos para la construcción del objeto de estudio han sido planteados por diversos autores, quienes han aportado diferentes concepciones epistemológicas. Precisamente en las líneas que preceden al texto citado de Bachelard, este autor ataca la opinión, y considera que uno de los primeros procedimientos de construcción de objeto es separarse de la opinión, así como Durkheim (1997) había sugerido alejarse de las prenociones, y Pierre Bourdieu, Passeron y Chamboredon (2008) hablan de mecanismos de ruptura con respecto al saber espontáneo. El verbo que utilizamos para denominar al acto de construir el objeto es objetivar, no en el sentido de hacer algo imparcial, sino en el de dar propiedades de objeto a fenómenos que, por sí mismos, no las tienen. Este proceso de construcción se evidencia en el establecimiento de un sistema de relaciones entre fenómenos, hechos, conceptos y problemas, y en la justificación de esas relaciones por parte del investigador; como resultado tenemos, entonces, objetos de estudio, no temas de investigación.

\section{Subnivel axiológico}

Como lo ha señalado Óscar Fernández (1992), los valores son concepciones de lo deseable, ideas que orientan las acciones en función de lo que se debe querer, de lo que se debe hacer. En el campo de la vida cotidiana, en general, los valores funcionan como puntos de partida de la acción; esto es, mucho del comportamiento social parte de lo que se considera deseable. Sin embargo, los valores también funcionan como metas, es decir, se aspira alcanzar determinado valor, o que otros lo alcancen. En el campo 
de la investigación socioeducativa y social en general, los valores igualmente funcionan como orientadores de la acción, en este caso, de la acción investigativa.

El lugar de los valores, desde el punto de vista de Max Weber (1973), es central en la investigación científico social, desde al menos dos vías: primero, "el tratamiento científico de los juicios de valor". Es menester recordar que Weber estaba muy interesado, en su teoría de la acción social, por comprender y explicar las acciones de los individuos en sociedad, y una de las formas para llevar a cabo este objetivo fue considerar que hay acciones sociales con arreglo a valores, es decir, que las acciones de las personas se pueden comprender y explicar si conocemos cuáles son los valores que motivan esa acción. Segundo, los valores están fuertemente ligados al proceso de investigación en cuanto a lo que Weber (1973) llamó "la orientación de nuestros intereses cognitivos" pues, según este autor, la selección de nuestros objetos de investigación no es objetiva, sino que está motivada por una serie de significaciones culturales, sociales, incluso morales que tienen sentido y son apreciadas por el sujeto que intenta conocer un fenómeno.

En la investigación científico-social y socioeducativa hay factores valorativos que nos mueve a investigar una cosa y no otra. Un ejemplo de esto es lo que Juan Luis Coraggio (1996) ha denominado investigación educativa legitimante y, por oposición, investigación educativa deslegitimante las cuales, según el autor, están orientadas por el objetivo de lograr la aceptación de políticas educativas, o bien, conseguir desprestigiarlas, pues su objetivo no es la búsqueda de la "verdad", sino de la razón a toda costa -como decía Arthur Schopenhauer (1996) - lícita o ilícitamente, y de acuerdo con lo que se considera deseable, ya sea por razones políticas, ideológicas, o por simple convencimiento. En todo caso y en cada una de tales razones, el punto de partida así como la aspiración es un ideal, o una valoración.

¿Cómo enfrentar el problema de los valores en la selección de nuestros objetos de investigación y de estudio? Norbert Elias, ha señalado al respecto: 
El problema que se plantea a los científicos sociales no puede solucionarse mediante una sencilla renuncia a las funciones de miembro de grupo en favor de las de investigador. Los científicos sociales no pueden dejar de tomar parte en los asuntos políticos y sociales de su grupo y su época, ni pueden evitar que estos les afecten. Además, su participación personal, su compromiso, constituyen una de las condiciones previas para comprender el problema que han de resolver como científicos. Pues, si bien para estudiar la estructura de una molécula no hace falta saber qué se sentiría si se fuese uno de sus átomos, para comprender las funciones de grupos humanos, es necesario conocer desde dentro cómo experimentan los seres humanos los grupos de los que forman parte y los que les son ajenos (1990: 28).

Lo planteado por Elias confirma la tesis de Max Weber sobre la necesidad de comprender los valores de los individuos que estudiamos $y$, por otro lado, sugiere también la necesidad de comprender nuestros propios valores, pues son estos los que orientan gran parte de nuestro trabajo investigativo. El problema radica, precisamente, en dejarnos llevar hacia una posición de "ingenuidad valorativa", no estar conscientes de nuestros propios dogmas, de nuestras consideraciones sobre lo deseable, pues si llegamos a ese punto de ingenuidad, la investigación puede verse demasiado sesgada por la pasión, es decir, por lo irracional.

En tanto podamos ser conscientes de nuestra posición axiológica, podremos tener claridad sobre los motivos de nuestras elecciones, de nuestros puntos de vista teóricos y de nuestras decisiones metodológicas. Solamente teniendo conciencia de ello, podemos, además, ser críticos con respecto a nuestros propios puntos de vista. Además, la conciencia de nuestros valores en el plano investigativo nos abre una puerta más, la de reconocer y estar abiertos y al tanto de posiciones ideológicamente contrarias de la que estamos desarrollando en el proceso de investigación.

\section{Nivel científico}

Etimológicamente, ciencia significa conocimiento, y llega a nuestra lengua, así como a muchos otros idiomas, por vía del latín (scientia). La ciencia es conocimiento resultante de la verificación empírica, y no solo del pensamiento libre, de la intuición o de la 
capacidad racional del sujeto. El científico no solo debe concebir teórico-racionalmente (abstracta y filosóficamente) sus objetos de estudio, lo cual es un imprescindible punto de partida, pero además debe verificar este empíricamente, sus enunciados, sus tesis iniciales, y obtener conclusiones que permitan repensar (confirmar, afinar, reformular o refutar) los principios teóricos de los que se ha partido. La clásica y siempre fundamental distinción kantiana entre pensar y conocer, resulta de gran utilidad para comprender la diferencia entre filosofía y ciencia:

Para conocer un objeto se requiere que yo pueda demostrar su posibilidad (ya sea por el testimonio de la experiencia, a partir de la realidad efectiva de él, ya sea a priori, por la razón). Pero pensar puedo pensar lo que quiera, con tal que no me contradiga a mí mismo, es decir, con tal que mi concepto sea un pensamiento posible, aunque yo no pueda asegurar que en el conjunto de todas las posibilidades a este (mi concepto) le corresponde, o no, un objeto. Pero para atribuirle a tal concepto validez objetiva (posibilidad real, ya que la primera era solamente la posibilidad lógica), se requiere algo más. Este algo más, empero, no precisa ser buscado en las fuentes teóricas del conocimiento; puede estar también en las prácticas (Kant, 2002: 92).

Del planteamiento kantiano se sigue que, lo pensable no es igual a lo cognoscible, el ser humano tiene la facultad de pensar más allá de lo que puede conocer. Por ejemplo, podemos pensar el alma, los dioses, la eternidad, todas las mitologías, pero estos fenómenos no los podemos conocer empíricamente, por tanto, no caben dentro del conocimiento científico. Se trata de fenómenos a los cuales nos podemos aproximar desde formas de conocimiento distintas, fenómenos de los que se encargan otros dominios, como al arte, la religión, la metafísica, pero no la ciencia.

Ahora bien, se podría contraargumentar que disciplinas como la historia o la filología, pueden ocuparse del estudio de las mitologías; sin embargo, estas disciplinas, en efecto, se ocupan de mitologías y otros temas similares, sin asumir, o sin el objetivo de comprobar la existencia o no, por ejemplo de las sirenas, de Odín o del minotauro, sino que, se encargan de estudiar los me- 
canismos culturales, históricos, sociales o estéticos (entre otros) de construcción de esas mitologías.

El nivel científico de la investigación social y educativa es el nivel de la verificación empírica. La principal distinción de la ciencia, con respecto a otras formas del conocimiento, es el interés, o la esencial tarea de contrastar ante la realidad, las ideas, las hipótesis, los enunciados, en fin, las argumentaciones. Este anhelo por la evidencia empírica, la verificación y la fundamentación del conocimiento sobre la experiencia, y no solamente sobre las ideas, es el resultado de la evolución del mismo conocimiento humano, y podríamos afirmar, que el germen de la verificación empírica está ya madurando en Aristóteles, y va a evolucionar y desarrollarse a cabalidad desde el materialismo aristotélico hasta el empirismo y positivismo del siglo XIX.

Aristóteles consideraba que las esencias están en las cosas, a diferencia de Platón (1988), quien pensaba que las esencias son formas ideales del ser, ideas que están en un cielo ininteligible que, como la luz del sol-según cuenta en su famosa alegoría de la caverna del capítulo sétimo de La República-, nos cegaría al verla directamente. Aristóteles, en cambio, considera que las esencias están en la cosa misma:

Tras esto, digamos que toda entidad se genera de algo del mismo nombre y esencia tanto las que son entidades naturales como las otras. Se generan, en efecto, o por arte, o por naturaleza, o por azar, o espontáneamente. Pues bien, el arte es un principio que está en otra cosa, mientras que la naturaleza es un principio que está en la cosa misma (en efecto, un hombre engendra a un hombre), y las restantes causas son, a su vez, privaciones de estas (1982: 474).

Entonces, para conocer las esencias hay que conocer las cosas. Por esto Durkheim (1997) ha sido enfático en su aforismo de "tratar los hechos sociales como cosas", pues al considerarlas objetos, podremos acercarnos a sus esencias. De este modo, debemos considerar también los hechos educativos como cosas, como objetos de estudio, sin olvidar lo sugerido por Elias en cuanto a la conciencia de nuestra relación con esos objetos. 
El objetivo de la investigación socioeducativa es entonces también verificar ideas, hipótesis, enunciados sobre la realidad educativa, en cuanto realidad social. El trabajo de verificación y de correspondencia con la realidad es lo que nos permite acceder al conocimiento de la realidad. En este sentido, la tesis de la verdad como correspondencia (Searle, 1997) cobra relevancia para los efectos investigativos, pues un enunciado sobre la realidad social y educativa es solo verdadero si corresponde con los hechos observables pues, si no hay correspondencia entre nuestras ideas y los hechos o los datos observables, nuestro enunciado se queda en el plano de la creencia, de las ideas, de la prenoción, pero no avanza al plano de las nociones científicas.

Una implicación importante de las ideas citadas es la necesidad de considerar el conocimiento científico como una de las representaciones posibles sobre la experiencia. Si la ciencia se basa en la experimentación y, tal como lo señalaba Kant (2002) "todo conocimiento inicia con la experiencia”, el procedimiento habitual es el de registrar, documentar, analizar y ofrecer una síntesis de la experiencia, pues esta no es necesariamente reproducible -no es repetible, pues hay experiencias que sí lo son- absolutamente. En todo caso, si la experiencia se pudiese reproducir tal cual es, y esta reproductibilidad significa que la podemos conocer, no haría entonces falta el conocimiento científico, pues la realidad estaría dada y sería transparente, bastaría con observarla para conocerla. Pero como necesitamos conocer una realidad que no es clara, que -como ha dicho Heráclito de Éfeso-, "ama esconderse" (Heráclito, 1900), entonces, la opción que nos queda es representar la realidad, esto es, transcribir la realidad por medio del lenguaje, en este caso, del lenguaje científico, solo que la realidad en su totalidad no es transcribible, lo que podemos es dar cuenta de una parte de la realidad social, aquella que es observable. Además, como en toda representación, y como en toda transcripción, es necesario acentuar algunos elementos y obviar otros, siendo entonces nuestro resultado parcial, a lo que Max Weber comenta:

La validez objetiva de todo saber empírico descansa en esto, y solo en esto: que la realidad dada se ordene según categorías que son subjetivas 
en un sentido específico, en cuanto representan el presupuesto de nuestro conocimiento y están ligadas al valor de aquella verdad que solo el saber empírico puede proporcionarnos (1973: 99).

Nuestras categorías de conocimiento, conceptos, y puntos de vista serán siempre parciales, y en tanto son parciales, son también subjetivos. Pero esta subjetividad se salva, al menos provisoriamente, a través de la verificación, de la correspondencia con la realidad y de la conciencia de nuestra posición en la estructura social o, como dice Pierre Bourdieu (2005), en el campo científico, con respecto a los fenómenos de los que intentamos dar cuenta. En última instancia, la única opción que tenemos es verificar y contrastar ante la realidad observable lo que pensamos, nuestras ideas y nuestros argumentos sobre la educación, el aprendizaje, el conocimiento, y el resto de fenómenos propios de la investigación socioeducativa.

\section{Nivel técnico}

La etimología nos enseña que la palabra técnica es una herencia griega que ha llegado a nuestro lenguaje y que proviene del vocablo $\tau \dot{\varepsilon} \chi v \eta$ (tékne), cuyo sentido es el de arte u oficio, o bien arte tanto como oficio al mismo tiempo. La técnica tiene como objetivo lograr un resultado específico y previamente concebido, no necesariamente fijado, pero sí previsto. Es por ello que un artista, un deportista, un educador, y también un investigador necesitan desarrollar una técnica que dé resultados en cada uno de sus respectivos campos disciplinares. Como se puede observar, la noción de técnica está entonces ligada a otras palabras como aplicación, práctica, objetivos, resultados. Y se espera que la técnica se adquiera, se desarrolle y se mejore conforme la práctica va indicándonos el camino.

La verificación empírica de la que hablamos en el apartado dedicado a la ciencia no es del todo arbitraria, pues se lleva a cabo mediante tres elementos fundamentales, la metodología, la operacionalización y la instrumentalización los cuales, en su conjunto, componen un nivel de aplicabilidad técnica, de trabajo de campo y de captación e incidencia sobre la realidad. 
La metodología (Corominas, 1983) es una palabra compuesta por tres voces griegas $\mu \varepsilon \tau \alpha$ (meta, más allá), ó $\delta o ́ \sigma$ (odos, camino),

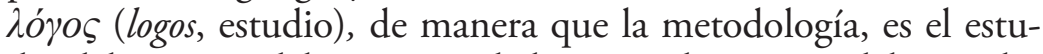
dio del camino, del proceso y de los procedimientos, del método, en este caso, investigativo (Beltrán, 2000). ¿Qué implicaciones mínimas tiene el estudio del método? Estudiar el método de investigación, no en general, sino particularmente en el campo de la investigación socioeducativa implica tener en consideración los métodos de las ciencias sociales, pues la mayor parte de los métodos con los que trabajamos en la investigación sobre procesos educativos, proviene de la sociología, la antropología, la psicología y del resto de lo que conocemos como ciencia social, de manera que la educación es el objeto, y el método es el científico-social. Lo importante acá es tener la capacidad, fruto de la experiencia y del estudio, de utilizar esos métodos para el objeto específico del que estamos tratando de dar cuenta.

La operacionalización, es un procedimiento muy concreto, y a la vez poco sencillo, pero fundamental en el proceso de investigación, pues consiste en un eslabón entre lo teórico-abstracto y lo empírico-concreto. D’ancona ha señalado sobre este asunto lo siguiente:

La operacionalización de conceptos teóricos constituye una fase intermedia en el proceso de investigación. Antecede al diseño de la indagación empírica siendo, a su vez, consecutivo a la formulación del problema de estudio. De la teoría (o marco teórico que conforma la investigación) se extraen unos conceptos y proposiciones. Los conceptos se traducen a términos operacionales. De ellos se deducen unas variables empíricas o indicadores que posibiliten la contrastación empírica del concepto que se analice. El término "operacionalización" -tomado de las ciencias naturales- es el que habitualmente se emplea para denotar los estadios implicados en el proceso de asignación de mediciones a conceptos (1996: 123).

Puede plantearse una definición quizás más acotada y significativa del proceso de operacionalización, diciendo que se trata del momento investigativo en el que definimos los elementos observables de la realidad que responden y corresponden, como evidencia, a las nociones teóricas, conceptos y constructos que estamos 
utilizando. ¿Qué conceptos son los que debemos operacionalizar? La respuesta sería: aquellos que necesitamos observar. Lo que no necesitamos observar, tampoco operacionalizar.

Evidentemente, como una investigación debe escribirse, esto es, debe utilizarse el lenguaje escrito, utilizaremos, por lo tanto, en nuestro estudio, muchas abstracciones, pero no es imprescindibles observar empíricamente todas, de modo que, por lo general, en nuestras preguntas de investigación y en nuestros objetivos, está lo que necesitamos observar. Por ejemplo, si algún investigador se propone analizar el tema del aprendizaje, necesita no solo decidir lo que entiende por este concepto, sino además, cómo se observa el aprendizaje en la realidad empírica, o para plantearlo en sentido inverso, qué elementos de la realidad observable corresponden a lo que el investigador entiende por aprendizaje.

Finalmente, en el proceso de invasión sobre la realidad, que es el plano técnico, necesitamos instrumentos (cuestionarios, tablas de observación, guías de entrevistas, tablas de cotejo, etc.) que están diseñados, específicamente, para cazar aquellos datos $\mathrm{u}$ objetos que perseguimos en nuestro proceso investigativo. $\mathrm{La}$ instrumentalización no es necesariamente antojadiza, sino funcional; debe funcionar para los propósitos propuestos, y diseñarse específicamente en función de esos propósitos. Los instrumentos tienen, como fin último, recolectar datos, de manera que deben estar ajustados con respecto a los que se pretende recolectar, y definidos a partir tanto del objeto de estudio como de las preguntas y objetivos de la investigación, en este caso se cumple la idea de que el método está condicionado por el objeto de estudio.

Es importante tener en cuenta también, las formas como se han utilizado los métodos y los instrumentos en los procesos investigativos reales, y no solo en los tratados de investigación, puesto que las debilidades de los tratados y los escritos sobre investigación (tal como este ensayo), es que presentan esquemas de cómo se hace investigación, sin hacerla, y solo refiriendo a la forma en que otros lo han hecho, pero resulta imprescindible el estudio directo de las formas mediante las cuales otros han aplicado los métodos, técnicas e instrumentos de investigación, tal como se sugiere en El oficio del sociólogo (Bourdieu, Passeron, Chamboredon, 2008: 11): 
El método — escribe Auguste Comte — no es susceptible de ser estudiado separadamente de las investigaciones en que se lo emplea; o, por lo menos, sería este un estudio muerto, incapaz de fecundar el espíritu que a él se consagre.

Como todo proceso técnico, no solo el estudio de lo que otros han hecho, sino también la experiencia propia resulta fundamental en el mejoramiento de la aplicabilidad. La utilización reiterada de un instrumento permite entender su lógica, sus características, y sus limitaciones.

Además, es necesario tomar en cuenta las necesidades intrínsecas del diseño del instrumento particular en el que estamos trabajando, esto es, los elementos que es necesario considerar en el momento de diseńo y de la aplicación del instrumento.

Igualmente, el momento de ejecución de este proceso instrumental depende de una cantidad considerable de variables, tales como la dinámica con los sujetos de investigación, lo que Taylor y Bogdan (1987) han llamado rapport, el contexto o las circunstancias de aplicación, las limitaciones del instrumento, entre otras. Con todo, la experiencia instrumental en la investigación, siempre tiene ese sabor a la aventura de adentrarse en la realidad social y educativa, sobre la que hemos reflexionado durante todo el proceso de diseño investigativo, y eso ya es una gratificación.

\section{El enfoque relacional para la investigación socioeducativa}

Desde Berkeley (1994), el tema de la relacionalidad del conocimiento es un asunto primordial en filosofía, en la teoría del conocimiento, y posteriormente en las ciencias sociales. En su Tratado sobre los principios del conocimiento humano publicado en 1710 (1994), Berkeley insiste en que lo que conocemos en realidad es el resultado del contacto entre nuestro aparato sensible y el mundo externo, de manera que nociones como sabor, color, olor, forma, no están ni en el objeto, ni en el sujeto, sino en el contacto entre ambos. Este enfoque es una concepción relacional de la realidad externa, la cual existe en el cuanto es percibida, es decir, la realidad existe cuando algún sujeto entra en contacto (relación) con ella. 
Si nos situamos no ya en el plano de la filosofía, sino en el de la ciencia, en este caso en el de las ciencias sociales, y con implicaciones para la investigación social y educativa, Pierre Bourdieu, en el texto titulado Pensar en términos relacionales, ha reformulado la famosa sentencia hegeliana de 1902 que dice Was vernünftig ist, das ist wirklich; und was wirklich ist, das ist vernünftig ${ }^{2}$ (Hegel, 2010: 11), cuando señala:

El modo relacional (en lugar del más estrechamente "estructuralista") de pensar, como lo demostró Cassirer (1923) en Substanzbegriff und Funktionsbegriff, es el sello de la ciencia moderna, y uno podría mostrar que yace detrás de empresas científicas al parecer tan distintas como las del formalista ruso Tinianov, el psicólogo social Kurt Lewin, Norbert Elias, y los pioneros del estructuralismo en antropología, lingüística e historia, desde Sapir y Jakobson hasta Dumézil y Lévi-Strauss. (Si ustedes lo verifican, comprobarán que tanto Lewin como Elias abrevan en Cassirer, como lo hago yo, para moverse más allá del sustancialismo aristotélico que impregna espontáneamente el pensamiento social.) Podría darle un giro a la famosa fórmula de Hegel y decir que lo real es lo relacional: lo que existe en el mundo social son las relaciones. No interacciones entre agentes o lazos intersubjetivos entre individuos, sino relaciones objetivas que existen "independientemente de la conciencia o la voluntad individual", como afirmó Marx (2005:150).

Este punto de vista sobre la realidad, es una concepción problematizadora del conocimiento, y de las representaciones que por medio de cualquier método del conocer, llevamos a cabo, pues se busca no solamente entender, comprender y explicar la realidad, sino además, captar las relaciones que dan forma a esa realidad social y educativa, relaciones que hacen de la realidad un fenómeno más complejo, pues no es solo una sustancia, una esencia, o un fenómeno, sino que se contempla como una lógica de relaciones.

En el texto Manifesto for a Relational Sociology, Mustafa Emirbayer (1997) ha puntualizado muy bien la distinción entre el pensamiento substancialista y el pensamiento relacional, mien-

\footnotetext{
${ }^{2}$ Lo que es racional es real; y lo que es real es racional (traducción propia).
} 
tras el primero observa y analiza la realidad en términos de seres, esencias, cosas, es decir, en términos de sustancias, el segundo se acerca al estudio de la realidad social y educativa en términos de relaciones.

Desde una perspectiva relacional, el todo es mayor que las partes, pues comprende o incluye las relaciones entre las partes, de manera que un enfoque analítico-sintético de la investigación socioeducativa observaría las partes, y luego presentaría una síntesis del análisis realizado, mientras que un enfoque relacional se concentraría en las relaciones entre las partes que componen el todo (la realidad socioeducativa o la unidad de análisis definida sobre esa realidad) sin prescindir del análisis de esas partes, ni de ofrecer una visión de conjunto y una síntesis del estudio realizado.

Por lo tanto, un enfoque relacional en términos investigativos, tiene implicaciones directas sobre la concepción del hecho de investigar en varios planos, de los cuales nos interesa puntualizar al menos tres concepciones sobre la investigación, que consideramos esenciales, y que podríamos resumir en los siguientes puntos:

3. Una concepción de los procesos de investigación: ya habíamos seńalado, parafraseando a Pierre Bourdieu y sus coautores (2008) que los actos investigativos no son cronológicos, son lógicos. Esta concepción de los procesos de investigación propone integrar los niveles formales de la investigación que estudiamos en el apartado anterior, lo filosófico, científico y técnico. De manera que una concepción relacional de la investigación pone especial atención y, valga decirlo, cuidado, al sistema de relaciones (lógica interna) intra-investigativas. No se trata, como a veces se cree, de seguir con rigidez una serie de pasos que van desde el planteamiento del tema, la justificación, el problema, los objetivos, etc., hasta la presentación del informe o de los resultados, como si fuesen cada uno de estos elementos partes sueltas o pasos aislados del proceso de investigación. Se trata, en cambio, de la construcción de un objeto de estudio, y de observar las relaciones lógicas entre este objeto y el resto de las partes formales del proceso de investigación. Estas relaciones lógicas se muestran $-y$ permiten alcanzar- especialmente en la coherencia necesaria 
entre el objeto de estudio y la pregunta de investigación, los componentes teóricos, metodológicos y los datos empíricos de los que se echará mano. En todo proceso de investigación social y educativa es necesaria una concepción de la realidad (subnivel ontológico), de la cual dependerá la idea que tengamos sobre la forma de conocer esa realidad, así como nuestra posición con respecto a ella. Todo lo anterior define el proceso de recolección de datos, pues habrá algunos que serán considerados válidos, mientras que otros no y, por último, esas concepciones definen también la forma en que analizamos los datos y las conclusiones que de ellos podamos obtener. Por ejemplo, si considero que la realidad es objetiva, y externa, mi forma de acercarme a ella será con una requerida distancia para no interferir con los datos, y trataré de analizar los datos para obtener conclusiones generales sobre la realidad, esta posición es un punto de vista positivista, mientras que si adopto otra postura, por ejemplo naturalista, partiré del principio de la relatividad de la realidad social, lo cual modificará mi distancia con respecto a los datos, y asimismo las conclusiones que obtenga de ellos, pues no tendré interés en generalizar mis resultados. Así, se puede apreciar que en toda investigación hay elementos filosóficos, científicos técnicos que tienen influencias recíprocas unos sobre otros.

4. Una concepción del objeto de estudio: desde un punto de vista relacional no hay temas de investigación, sino objetos de estudio, y el objeto es una construcción del investigador. Ya Bachelard ha dicho que "los problemas no se plantean a sí mismos... nada está dado, todo se construye" (2000: 16). La construcción del objeto es uno de los procedimientos fundamentales del proceso investigativo, desde el punto de vista relacional, tal como lo señala Pierre Bourdieu cuando, al considerar la importancia de los principios del pensamiento relacional dice: "En ningún campo se aplican mejor estos principios que en la construcción del objeto, la operación sin duda más importante $y$, sin embargo, más ignorada, en particular en la tradición dominante, que se organiza en torno a la oposición entre la 'teoría' y la 'metodología'” (2005: 167). Efectivamente, aunque no contamos con una estadística preci- 
sa, pocas obras o manuales de investigación se refieren al proceso epistemológico de la construcción del objeto de estudio, y como la realidad nos mueve a pensar en términos de unidades y de realidades -tal como la realidad se nos presenta- no es habitual pensar en términos de relaciones, pero es fundamental en el sentido de que la investigación en ciencias sociales y de la educación no se ocupa de objetos brutos, sino de sistemas de relaciones. Por ejemplo, la exclusión educativa, la desigualdad, o el aprendizaje, son fenómenos cuya existencia depende de relaciones. No hay un aprendizaje como término absoluto y autocontenido, sino que son los individuos los que aprenden, y esto tiene que ver con la posición que ocupan en el espacio social, y sus relaciones, tanto consigo mismos como con los otros, con los contenidos que se estudian, y con una cantidad de variables a veces difícil de precisar. Por lo tanto, la tarea del investigador es delinear y decidir cuáles son los elementos que constituyen ese sistema de relaciones pero, sobre todo, justificar y explicar las relaciones entre esos elementos, los cuales pueden ser fenómenos, conceptos, problemas, variables, entre otros.

5. Una concepción de la realidad: la realidad social y educativa se convierte, desde este punto de vista, en un espacio de relaciones, las que, si bien existen por los sujetos, no están en los sujetos, sino en sus prácticas y, sobre todo, en sus contactos e interacciones con otros y con su espacio social. Esta concepción de la realidad, que no es sustancialista, orienta entonces la búsqueda de la explicación, la causalidad, y la comprensión de los fenómenos socioeducativos, haciendo el énfasis en las relaciones.

Estos tres elementos centrales del enfoque relacional en términos investigativos ofrecen un gran potencial para la investigación socioeducativa, tanto en el plano de las herramientas de investigación como en el plano de la orientación de las investigaciones sobre los fenómenos educativos.

Uno de los aspectos centrales que debemos tomar en cuenta es que la investigación educativa tiene particularidades en cuanto se trata de un espacio de convergencia entre una cantidad de variables muy grande, es un fenómeno social de alta compleji- 
dad, multideterminado, además es un espacio de luchas, donde confluyen elementos políticos, sociales, económicos, culturales, procedimentales, psicológicos, y muchos más.

Quizá nunca podamos dar cuenta de la educación, ni de la realidad como una totalidad, pero el enfoque relacional permite acercarse a entender las lógicas de las relaciones que constituyen elementos importantes del fenómeno socioeducativo. En los siguientes cinco puntos, ensayaremos las posibilidades que el enfoque relacional plantea a la investigación educativa, si bien este esfuerzo es relativamente general, se toman en cuenta la integración de los niveles formales de investigación para el estudio de los fenómenos educativos:

1. La integración de los niveles investigativos: el principio de integración de los niveles investigativos es más que necesario y útil en los procesos de investigación educativa, pues al ser la educación un fenómeno tan presente en todas las sociedades, y de tanta influencia sobre los individuos a partir de una multiplicidad de experiencias, tenemos como resultado que la mayor parte de las personas tienen una concepción sobre los fenómenos educativos. Puede ser importante contar con estas diversas voces sobre la educación, pero para efectos investigativos, este fenómeno dificulta la sistematización de los procesos. De manera que un enfoque relacional interno, que centra la atención en las relaciones lógicas de las partes del proceso de investigación socioeducativa, permite controlar (no necesariamente eliminar) los sesgos que la opinión y el sentido común ocasionan sobre los sujetos cognoscentes en su proceso de construcción de conocimiento a partir de la investigación. Esta integración de los niveles investigativos permite al investigador tener conciencia de sus propias concepciones filosóficas, científicas y técnicas $\mathrm{y}$, sobre todo, hacer visibles y conscientes las relaciones que se están planteando entre cada uno de los niveles de investigación. En un terreno como el educativo, en el que se cruzan valores, posturas filosóficas, científicas, condiciones históricas, culturales, económicas y sociales, tener conciencia de la propia postura como investigador, permite mayor control sobre los puntos de vista sesgados que podamos tener con respecto a la educación. 
2. La educación como "campo" de relaciones (luchas): en la educación como fenómeno aplica muy bien la noción de campo planteada por Pierre Bourdieu (2005), pues se trata de un espacio social en el que existen diferentes relaciones, luchas y disputas por el capital que está en juego. El problema del fenómeno educativo es que existen muchos capitales en juego, pero sobre todo, dos de los más importantes, el capital cultural y el capital económico, en diferentes formas y con una distribución desigual. Al tener estos dos capitales como centro de las relaciones y las tensiones de los actores involucrados en el campo, las luchas son constantes e implican distintas formas de participación. Las luchas son diferenciadas, pero normalmente la esencia de estas disputas está centrada en el tema de los recursos materiales necesarios para el desarrollo de buenas prácticas educativas, la disminución de la exclusión social que está presente en la educación, el tratamiento de la pobreza y la desigualdad, también implicadas en la educación.

3. El análisis de redes socioeducativas: el enfoque de redes sociales presenta diversas posibilidades de análisis de la educación, a pesar de que ha sido, al menos para el caso costarricense, poco utilizado en términos investigativos (Morales, 2011). El análisis de redes sociales es un tipo de análisis relacional en el que se busca dar cuenta de las interacciones entre individuos y también entre actores tales como grupos, pares, instituciones, entre otros. Uno de los aportes del análisis de redes sociales a la investigación educativa es que permite observar la fuerza que tienen ciertos lazos en el desarrollo de la educación, y muy puntualmente en fenómenos como el aprendizaje, la actitud hacia el estudio, la toma de decisiones en materia de política educativa y de gestión de la educación. Esta forma de estudio busca hacer visibles las redes que existen en la realidad (no en la realidad virtual, en la cual las redes ya son visibles, aunque muchas veces son solo virtuales y no tan reales), pero que no son observables con facilidad. A partir de la observación de estas redes es posible determinar la influencia que tienen sobre las conductas individuales, las posiciones que ocupan los individuos en una red, y la fuerza de lo social en lo educativo. 
4. Micro y macro relacionalidad de los fenómenos educativos: en la educación confluyen o se manifiestan tantos actores y variables, que una forma de acercarse al problema educativo es estudiar la relacionalidad a nivel macro y micro, y al mismo tiempo tratar de entender las relaciones entre esos dos niveles de relacionalidad. Podríamos sostener que a nivel individual, existe una serie de relaciones cotidianas con gran influencia sobre los sujetos, su comportamiento, sus actitudes hacia la educación y sobre su desempeño educativo. De hecho, es necesario partir de una concepción novedosa que podríamos llamar "aprendizaje relacional", que implica la capacidad, cultivada por el sujeto, de establecer relaciones entre datos, conceptos, ideas, problemas, con el fin de comprender su entorno, y la realidad a la cual se está enfrentando. Este es un nivel de micro-relacionalidad, tanto el aprendizaje, como las interacciones a lo interno del salón de clase, la familia, y los grupos de pares. Otro nivel que podríamos llamar de macrorelacionalidad, es el que se da en el plano de las decisiones políticas y las condiciones histórico-sociales, entendidas igualmente como sistemas de relaciones que afectan directamente las cotidianidades de los individuos en cuanto a sus procesos educativos. Wright Mills (1961) había llamado imaginación sociológica a la capacidad de establecer, entender y comprender las relaciones entre biografía e historia, es decir entre el curso individual, y el de la estructura social en movimiento, en devenir. De manera que, entender las relacionalidades a nivel micro y a nivel macro social, así como la interacción entre esos dos niveles, es una forma de acercarse a los fenómenos educativos que puede ser potencialmente fecunda, pues mucho de lo que pasa en el aula, de lo que ocurre en la familia, y de lo que sucede con el aprendizaje y el desarrollo de los estudiantes, está condicionado por relaciones e interacciones a esos dos niveles y entre esos dos niveles.

5. La educación como un bien relacional: la educación ha sido históricamente, y sigue siéndolo en la actualidad, objeto de críticas por sus esquemas rígidos, por su arbitrariedad, y por su jerarquización. Pierre Bourdieu (1990) se ha referido a la educación y a las acciones pedagógicas con actos de vio- 
lencia simbólica, de inculcación, y Michel Foucault (1983) ha planteado que la institución escolar es una institución de asilo, con la misma lógica de las cárceles. Esto implica que la educación ha sido contemplada como un bien unidireccional, que unos sujetos poseen y que otros sujetos reciben, igual que la educación bancaria de Paulo Freire (2005). Desde la perspectiva de Pierpaolo Donati, existen bienes relacionales, los cuales son definidos de la siguiente manera:

It is important to emphasize that from the beginning that the type of goods that we call relational cannot be traced back to traditional or postmodern forms of social organization, because they require conditions that only modernity has created by making individuals more free and guaranteeing a considerable amount of social mobility. For a long time these goods have been dismissed or even repressed by capitalistic society as well as by societies dominated by dictatorships. Today they are emerging as the yeast of an advanced democracy. They are created precisely where relations between consociates are tendentially symmetrical (not hierarchical), free and responsible (not conditioned by authoritative norms or powers) not mercantile, in the sense of being dictated by pursuit of individual profit. ${ }^{3}$ (2015: 198).

Por tanto, considerar la educación como un bien relacional, podría ser una forma de superar las nociones tradicionales de acercarse al fenómeno educativo, tan cargadas de autoritarismo, arbitrariedad, control y poder. Y quizás podría ser también una forma de superar lo que Francois Dubet (2007) ha denominado las "mutaciones y declive de la institución escolar", para caracterizar la institución escolar en nuestro tiempo, una época en la cual los valores tradicionales asociados a la educación (en la que

\footnotetext{
${ }^{3}$ Es importante destacar que, desde el principio, el tipo de bienes que llamamos relacionales no se remontan a las formas tradicionales o posmodernas de organización social, ya que requieren condiciones que solo la modernidad ha creado al hacer a los individuos más libres y garantizando una cantidad considerable de movilidad social. Durante mucho tiempo estos bienes han sido ignorados o incluso reprimidos por la sociedad capitalista, así como por las sociedades dominadas por dictaduras. Hoy en día se están convirtiendo en la levadura de una democracia avanzada. Son creados precisamente donde las relaciones entre los con-socios son tendencialmente simétricas (no jerárquicas), libres y responsables (no condicionadas por normas o poderes autoritarios) no mercantiles, en el sentido de ser dictada por búsqueda del beneficio individual (traducción propia).
} 
se consideraba a la institución escolar como una especie de templo del conocimiento, y al docente como el portador de la luz de la sabiduría) están en crisis, y la institución escolar afronta un problema de legitimidad asociado, además, a los problemas estructurales, al menos para América Latina, de pobreza, exclusión y desigualdad en el campo educativo. Si consideramos la educación como un bien relacional, en el que todas las partes tienen beneficios y responsabilidades, pero las relaciones son simétricas y no autoritarias, podríamos estar frente a una respuesta a los problemas educativos que enfrentamos en la actualidad. Sin embargo, esto es teórico, es tan solo un aporte del enfoque relacional de la investigación educativa y, entonces, se trata de investigar las posibilidades de concebir la educación como un bien relacional, con las características mencionadas.

\section{CONSIDERACIONES FINALES}

El estudio de la investigación educativa plantea desafíos en lo formal, es decir, en cuanto a los niveles presentes en la investigación, los protocolos y, en general, en cuanto a las formas de hacer y pensar la investigación, y también en cuanto a lo sustantivo, esto es, la forma como se considera la educación y por lo tanto las concepciones que tenemos acerca de ella, así como las formas de estudiarla.

Una primera conclusión es que cada nivel de investigación es el fundamento de las partes que integran un diseńo investigativo, y a pesar de que no están presentes como parte o como apartados específicos y explícitos del diseño, constituyen la base sobre la cual se erige el diseño.

Partiendo de una versión elemental de los elementos que conforman un diseño de investigación, sostenemos que la construcción de un problema y su justificación, tiene que ver con el nivel filosófico de la investigación, pues implica una concepción de la realidad y además una idea de cómo se puede conocer la realidad, esto es lo que desarrollamos en cuanto a lo ontológico y lo epistemológico.

Posteriormente, la construcción teórica, el marco de referencia es un asunto científico y también filosófico, pues se trata de 
construir una argumentación en el plano de las ideas, de los conceptos y de los constructos que se utilizarán luego en la etapa de verificación científica. Ahora bien, hay teorías o argumentaciones que pueden provenir de la filosofía, es decir, que no necesariamente han sido verificadas con anterioridad a nuestro estudio, o que pueden venir de distintos campos científicos, y que ya han sido estudiadas en el plano de la verificación.

En la etapa de recolección y análisis de los datos estamos ante un nivel científico y técnico, pues se trata de verificar nuestras hipótesis y nuestras ideas en el campo, contrastarlas con la realidad observable, analizar la correspondencia y la significación de los datos observados para luego aportar una perspectiva final, al menos para nuestro estudio, a sabiendas que esa perspectiva es la final pero no la última, pues es momentánea.

El subnivel filosófico, que habíamos denominado axiológico, está presente en todo el trabajo; lo importante es tener conciencia de nuestra posición, de distancia o de compromiso con respecto a la investigación que estamos realizando, con respecto a nuestro objeto de estudio y nuestros datos, con el fin de controlar lo más posible el sesgo que nuestra subjetividad puede hacer intervenir en el proceso investigativo.

En general, los tres niveles analizados, y el enfoque relacional, aportan a la investigación educativa rigor, no rigidez, y además, una concepción de lo que significa hacer investigación en el campo de la educación entendida como una ciencia social, es decir, que busca dar cuenta de fenómenos en los que estamos de una $\mathrm{u}$ otra forma involucrados. Como Norbert Elias lo ha sugerido, es importante tener claro que nuestro objetivo como investigadores, no es más que este:

El objetivo general del trabajo científico es el mismo en ambos campos; despojado de su corteza filosófica, consiste en descubrir cómo y por qué se relacionan entre sí los fenómenos registrados. Pero, a diferencia de las ciencias de la naturaleza, que buscan aclarar las relaciones planteadas entre fenómenos prehumanos, las ciencias sociales estudian relaciones interpersonales. Aquí el ser humano se encuentra consigo mismo y con los demás; los objetos son, al mismo tiempo, sujetos. La tarea del científico social es comprender, y hacer que los demás comprendan, cambiantes conjuntos 
de interrelaciones formadas por los seres humanos, la naturaleza de esos lazos y la estructura de esos cambios (1990: 23).

Elias pone especial interés en la importancia de lo relacional, y en la necesidad de comprender y hacer comprensibles los fenómenos sociales, o para nuestro interés, socioeducativos, lo cual corresponde y fundamenta nuestra propuesta, en el sentido de la importancia que otorgamos desde nuestra perspectiva a la claridad sobre los niveles involucrados en los procesos investigativos, las relaciones entre estos niveles y la relevancia que tienen para todo diseño de investigación y sobre todo para la rigurosidad investigativa.

Además, es el interés de este trabajo plantear una manera de observar la educación, entendiendo el fenómeno educativo como un campo de relaciones, y como un bien o un capital relacional, lo cual sugiere una postura no solo educativa, sino también investigativa. Esto podríamos considerarlo un enfoque de investigación que tiene como objetivo, mediante el realce de los elementos relacionales de la educación, y de la noción de bien relacional, servir como punto de partida para investigar distintas partes que componen la realidad socioeducativa, con el fin de mejorar tanto la comprensión que tenemos del fenómeno, como el fenómeno en sí.

\section{REFERENCIAS BIBLIOGRÁFICAS}

Aristóteles. Metafísica, $2^{\circ}$ ed. rev., Madrid, Gredos, Biblioteca Clásica, 1982.

Bachelard, G. La formación del espiritu cientifico, $23^{\circ}$ ed., México, Siglo XXI Editores, 2000.

Beltrán, Miguel. Perspectivas sociales y conocimiento, Barcelona, Anthropos, 2000.

Berkeley, George. Tratado sobre los principios del conocimiento humano, Barcelona, Editorial Altaya, 1994. (Grandes obras del pensamiento, núm. 35)

Bourdieu, P., y Passeron, J. C. Reproduction in education, society and culture, vol. 4, California, Sage, 1990. 
Bourdieu, P. Una invitación a la sociología reflexiva, México, Siglo XXI Editores, 2005.

Bourdieu, P. Passeron, J. P. y Chamboredon, J. P. El oficio de sociólogo, México, Siglo XXI Editores, 2008.

Coraggio, J. L. "Sobre la investigación y su relación con los paradigmas educativos, en Novas politicas educacionais: criticas e perspectivas, 1998.

Corominas, J. Diccionario critico etimológico castellano e hispánico, Joan Corominas, José A. Pascual, 5 vols., Madrid, Gredos, 1983.

D’ancona, M. Á. C. Metodología cuantitativa: estrategias y técnicas de investigación social, Madrid, Síntesis, 1996.

Donati, Pierpaolo. The Relational Subject, United Kingdom, Cambridge University Press, 2015.

Durkheim, E. Las reglas del método sociológico, vol. 86, Ediciones Akal, 1997.

Dubet, F. "El declive y las mutaciones de la institución", en $R e-$ vista de Antropología social, núm. 16, 2007, pp. 39-66.

Elias, N. Compromiso y distanciamiento. Ensayos de Sociología del conocimiento, Barcelona, Península, 1990, 63-65.

Emirbayer, M. "Manifesto for a relational sociology 1", en American journal of sociology, vol. 103, núm. 2, 1997, pp. 281-317.

Fernández, Oscar. "¿Qué valores valen hoy en Costa Rica?”, en Villasuso Estomba, Juan Manuel (Ed.), El nuevo rostro de Costa Rica, Costa Rica, CEDAL, 1992.

Foucault, M. Vigilar y castigar: nacimiento de la prisión, México, Siglo XXI Editores, 1983.

Freire, P. Pedagogía del oprimido, México, Siglo XXI Editores, 2005.

Hegel, G. W. F. Grundlinien der philosophie des rechts (ohne fussnoten), Signed by Cosmoedu.com, 2010.

Heráclito. Sobre la naturaleza: doxografía y fragmentos completos, San Pedro de Montes de Oca, C. R., Universidad de Costa Rica, Facultad de Letras, Escuela de Filosofía, 1900.

Jaeger, Werner. Paideia: los ideales de la cultura griega, $2^{\circ}$ ed., México, Fondo de Cultura Económica 1962.

Kant, Immanuel. Crítica de la razón pura, trad. J. Rovira Armengol, Barcelona, Ediciones Folio, 2002. 
Láscaris Comneno, Constantino. Palabras, San José, Costa Rica, Fernández-Arce, 1976.

Mills, C. W. La imaginación sociológica (Vol. 2). Fondo de Cultura Económica, 1961.

Morales Zúñiga, Luis Carlos. "Análisis de redes sociales como posibilidad teórico-metodológica para la investigación educativa", en Revista Actualidades Investigativas en Educación, vol. 11, núm. 3, Costa Rica, UCR, Instituto de Investigación en Educación, 2011.

Nietzsche, F. "Sobre verdad y mentira en sentido extramoral", en Revista Teorema, Valencia, 1980.

Platón. República, Madrid, Gredos, Biblioteca Clásica, 1988.

Sábato, E. Uno y el universo, Buenos Aires, Ed. Sudamericana, 1945.

Sampieri, R. H., Collado, C. F., Lucio, P. B., y Pérez, M. D. L. L. C. Metodología de la investigación, McGraw-Hill, 1998.

Searle, J. R. La construcción de la realidad social, Barcelona, Buenos Aires, México, Paidós, 1997.

Schopenhauer, A. El arte de tener razón: expuesto en 38 estratagemas, vol. 208, Argentina, Edaf, 1996.

Taylor, S. J., y Bogdan, R. Introducción a los métodos cualitativos de investigación, Barcelona, Buenos Aires México, Paidós, 1987.

Weber, Max. Ensayos sobre metodología sociológica, Buenos Aires, Amorrotu Editores, 1973.

Weber, M. El politico y el científico, España, Alianza Editorial, 5० ed., El libro de bolsillo, 181, 1979. 